\title{
Thermodynamics of the Schwarzschild and Reissner-Nordström black holes under the Snyder-de Sitter model
}

\author{
H. Hassanabadi ${ }^{1}$, E. Maghsoodi ${ }^{4,5}$, Won Sang Chung ${ }^{2}$, M. de Montigny ${ }^{3, a}$ \\ ${ }^{1}$ Faculty of Physics, Shahrood University of Technology, Shahrood 3619995161-316, Iran \\ ${ }^{2}$ Department of Physics and Research Institute of Natural Science, College of Natural Science, Gyeongsang National University, Jinju 660-701, \\ Korea \\ ${ }^{3}$ Faculté Saint-Jean, University of Alberta, Edmonton T6C 4G9, Canada \\ ${ }^{4}$ Department of Physics, Faculty of Science, Lorestan University, Khoramabad, Iran \\ ${ }^{5}$ Research Institute for Astronomy and Astrophysics of Maragha (RIAAM), P.O. Box: 55134-441, Maragha, Iran
}

Received: 21 June 2019 / Accepted: 5 November 2019 / Published online: 18 November 2019

(C) The Author(s) 2019

\begin{abstract}
This paper examines the effects of a new form of the extended generalized uncertainty principle in the Snyderde Sitter model on the thermodynamics of the Schwarzschild and Reissner-Nordström black holes. Firstly, we present a generalization of the minimal length uncertainty relation with two deformation parameters. Then we obtain the corrected mass-temperature relation, entropy and heat capacity for Schwarzschild black hole. Also we investigate the effect of the corrected uncertainty principle on the thermodynamics of the charged black holes. Our discussion of the corrected entropy involves a heuristic analysis of a particle which is absorbed by the black hole. Finally, we compare the thermodynamics of a charged black hole with the thermodynamics of a Schwarzschild black hole and with the usual forms, that is, without corrections to the uncertainty principle.
\end{abstract}

\section{Contents}

1 Introduction ............... . . 1

2 The new extended uncertainty principle . . . . . . 2

3 Thermodynamics of Schwarzschild black hole . . . 2

4 Thermodynamics of Reissner-Nordström black hole 4

5 Conclusions ............. 6

References.............. 6

\section{Introduction}

The consideration of the fundamental length scale at the order of the Planck length leads to a nonzero minimal uncertainty in the measurement of the position. In high energy physics, the study of deformed quantum mechanics plays a crucial role in removing infinities in standard field theories. In the

a e-mail: mdemonti@ualberta.ca last decade, some approaches towards understanding physics at this scale, based either on string theory or other quantum gravity paradigm have been studied [1-7].

The modified commutation relations between position and momenta, commonly known as Generalized Uncertainty Principle (GUP) and other similar relationships, are related to the prediction of a minimum measurable length [8-16]. This approach is motivated by the investigation of the quantum behaviour of black holes as the most interesting objects in physics. The study of black hole physics determined that a valid theory of quantum gravity must include a minimum length scale. Therefore the energy in any region of space below the Planck length is greater than the energy required to create a mini black hole in that region of space. Furthermore, some authors have recently proposed the corrected uncertainty principle which also implies the existence of a maximum measurable momentum [17]. Actually, because this GUP is consistent with Doubly Special Relativity theories (DSR) [18-22], this GUP implies the existence of a minimum measurable length while the existence of a minimum measurable momentum in turn requires the modification of the Heisenberg uncertainty principle into an Extended Uncertainty Principle (EUP) [23-30]. The more General Extended Generalized Uncertainty Principle (EGUP) is obtained by combining GUP and EUP, from a model of DSR on an (anti)de Sitter background. Actually, the connection between GUP and EUP is revealed with EGUP [31-35]. In Ref. [32], the deformed two-dimensional relativistic bosonic oscillator equation for charged spin 0 and spin 1 particles moving in a uniform magnetic field with the Snyder-de Sitter model has been studied.

The better-motivated derivations of the EGUP are given as GUP and EUP that motivated by the quantum-gravity idea 
of the smallest possible position and by physics in anti-de Sitter space respectively. Compared with the GUP and EUP, the EGUP can be applied to a large scale spacetime, hence its applicability range is wider. By defining the concise calculation method and understanding the physical meaning, it provides a reference for the determination of the thermodynamical properties of the black holes. In recent years, the study of the Schwarzschild black hole physics with GUP [36-40] has revealed that the Hawking radiation of a black hole depends on the properties of the black hole and the tunneling particle. In Ref. [30], an effective EUP-corrected Schwarzschild metric is constructed, and the associated black hole characteristics are considered. In Ref. [41] the thermodynamics of Schwarzschild and Reissner-Nordström (RN) black holes in the framework of GUP with correction terms up to fourth order in momentum uncertainty have been investigated and this has led to the existence of a remnant mass thereby preventing the complete evaporation of the black hole. Also it has been observed that analytical expressions for these masses can be obtained for terms of the order of $(\Delta p)^{8}$ in the momentum uncertainty.

In Ref. [42], the effect of space noncommutativity on the thermodynamics of RN charged black hole is investigated. Also, the Gauss-Bonnet theorem (GBT) is examined with quantum corrections through EUP and the corresponding deflection angle is obtained [43]. In Ref. [25], the influence of the GUP and EUP on the thermodynamics of the FRW universe has been investigated. In this paper, we have studied the thermodynamic properties of the Schwarzschild and RN black holes in the framework of EGUP with two deformation parameters.

This paper is organized as follows: in Sect. 2 , we present the new form of EGUP in Snyder-de Sitter model that we consider. In Sect. 3, we calculate the corrected masstemperature relation and heat capacity and entropy for the Schwarzschild black hole. In Sect. 4 by considering the RN charged black hole, we discuss the EGUP corrected thermodynamic properties. Finally, a conclusion is presented in Sect. 5. Before proceeding any further, it should be noted that in this manuscript, we set $\hbar=1$.

\section{The new extended uncertainty principle}

In the non-relativistic Snyder-de Sitter model, we introduce the deformed Heisenberg algebra in three-dimensional case by the following commutation relation as [32]

$$
\begin{aligned}
& {\left[X_{i}, P_{j}\right]=i\left(\delta_{i j}+\alpha_{1} X_{i} X_{j}+\alpha_{2} P_{i} P_{j}\right.} \\
& \left.\quad+\sqrt{\alpha_{1} \alpha_{2}}\left(X_{i} P_{j}+P_{i} X_{j}\right)\right), \quad i=1,2,3,
\end{aligned}
$$

where $\alpha_{1}, \alpha_{2}$ are the small positive parameters of deformation. We define $L_{k}$ as the component of the angular momen- tum, which can be expressed as follows

$L_{k}=\varepsilon_{i j k} X_{i} P_{j}$,

satisfying the usual algebra

$$
\begin{gathered}
{\left[X_{i}, X_{j}\right]=i \alpha_{2} \varepsilon_{i j k} L_{k}, \quad\left[P_{i}, P_{j}\right]=i \alpha_{1} \varepsilon_{i j k} L_{k}} \\
{\left[L_{i}, X_{j}\right]=i \varepsilon_{i j k} X_{k}, \quad\left[L_{i}, P_{j}\right]=i \varepsilon_{i j k} P_{k},} \\
{\left[L_{i}, L_{j}\right]=i \varepsilon_{i j k} L_{k} .}
\end{gathered}
$$

According to quantum mechanics, inserting the commutation relations (2.3) into Eq. (2.1) for one-dimensional case leads to

$$
\begin{aligned}
& \Delta X \Delta P \geqslant \frac{1}{2}\left(1+\chi+\alpha_{1}(\Delta X)^{2}+\alpha_{2}(\Delta P)^{2}\right. \\
& \left.-2 \sqrt{\alpha_{1} \alpha_{2}} \Delta X \Delta P\right),
\end{aligned}
$$

where $\chi=\left(\sqrt{\alpha_{1}}\langle X\rangle+\sqrt{\alpha_{2}}\langle P\rangle\right)^{2} \geqslant 0$. The relation (2.4) implies the appearance of a nonzero minimal length in position and momentum uncertainties [32]

$$
\begin{aligned}
& \Delta X \geqslant \sqrt{\frac{\alpha_{2}(1+\chi)}{1+2 \sqrt{\alpha_{1} \alpha_{2}}}} \\
& \Delta P \geqslant \sqrt{\frac{\alpha_{1}(1+\chi)}{1+2 \sqrt{\alpha_{1} \alpha_{2}}}} .
\end{aligned}
$$

By defining $c$ as the speed of light and $\kappa$ as the Boltzmann constant, we can consider the black hole thermodynamics for any massless quantum particle near the black hole horizon with mass $M$ as follows [29]

$T=\frac{c \Delta P}{\kappa}$,

and by inserting the obtained minimal momentum as Eq. (2.5) into Eq. (2.6), the lower bound for the black hole temperature has been found as

$T \geqslant T_{\min }=\frac{c}{\kappa} \sqrt{\frac{\alpha_{1}(1+\chi)}{1+2 \sqrt{\alpha_{1} \alpha_{2}}}}$,

\section{Thermodynamics of Schwarzschild black hole}

We utilize the Schwarzschild radius of the black hole as $r_{S}=$ $\frac{2 G M}{c^{2}}$ [41] where $G$ is the Newton's universal gravitational constant and we can consider the position uncertainty of a particle as the order of the Schwarzschild radius of the black hole near the horizon of the black hole

$\Delta X=\lambda r_{S}=\frac{2 \lambda G M}{c^{2}}$,

where $\lambda$ is a scale factor. By substituting Eqs. (2.6) and (3.1) into Eq. (2.4) we find

$$
\frac{2 \lambda \kappa G M T}{c^{3}}=\frac{1}{2}\left(1+\chi+\alpha_{1}\left(\frac{2 \lambda G M}{c^{2}}\right)^{2}\right.
$$




$$
\left.+\alpha_{2}\left(\frac{\kappa T}{c}\right)^{2}-2 \sqrt{\alpha_{1} \alpha_{2}}\left(\frac{2 \lambda \kappa G M T}{c^{3}}\right)\right) .
$$

By solving above equation versus $T$ and by introducing $m_{P}$ as the Planck mass, we have obtained the following solution for the black hole mass

$$
\begin{aligned}
M= & \frac{1}{8 G^{2} \lambda^{2} \alpha_{1}}\left\{4 c \kappa \lambda G T\left(1+\sqrt{\alpha_{1} \alpha_{2}}\right)\right. \\
& \left.-\sqrt{-16 G^{2} \lambda^{2} \alpha_{1}\left(c^{4}(1+\chi)+c^{2} \kappa^{2} T^{2} \alpha_{2}\right)+(4 c \kappa \lambda G T)^{2}\left(1+\sqrt{\alpha_{1} \alpha_{2}}\right)^{2}}\right\} .
\end{aligned}
$$

of the absence EGUP is comparable with the semiclassical temperature obtained as $T=\frac{(m P c)^{2}}{8 \pi \kappa M}$ in Ref. [29].

In the usual case, the heat capacity for the black hole is given by

$C=c^{2} \frac{d M}{d T}$.

Then, by considering the mass-temperature relation given in Eq. (3.3), the heat capacity corresponding to the uncertainty relation that arises from EGUP black hole can be obtained as

$C=\frac{c^{3} \kappa\left(-2 c \kappa \pi G T\left(1+2 \sqrt{\alpha_{1} \alpha_{2}}\right)+\left(1+\sqrt{\alpha_{1} \alpha_{2}}\right) \sqrt{-4 c^{2} \pi^{2} G^{2}\left(c^{2}(1+\chi) \alpha_{1}-\kappa^{2} T^{2}\left(1+2 \sqrt{\alpha_{1} \alpha_{2}}\right)\right)}\right)}{4 \pi G \alpha_{1} \sqrt{-4 c^{2} \pi^{2} G^{2}\left(c^{2}(1+\chi) \alpha_{1}-\kappa^{2} T^{2}\left(1+2 \sqrt{\alpha_{1} \alpha_{2}}\right)\right)}}$.

For small values of deformation parameters, the above equation can be written as

$M=\frac{c^{4}(1+\chi)+\alpha_{2} c^{2} \kappa^{2} T^{2}}{4 c \kappa \lambda G T\left(1+\sqrt{\alpha_{1} \alpha_{2}}\right)}$.

With $\alpha_{1}=\alpha_{2}=\chi=0$ in the absence of correction due to EGUP and considering the relationship of the Planck mass as $\left(m_{P} c\right)^{2}=\frac{c^{3}}{G}$, we have

$M=\frac{c^{4}}{4 c \kappa \lambda G T}=\frac{\left(m_{P} c\right)^{2}}{4 \kappa \lambda T}$.

If we compare this expression with the semiclassical mass $M=\frac{\left(m_{P} c\right)^{2}}{8 \pi \kappa T}$ [42], we can obtain $\lambda=2 \pi$. By solving Eq. (3.2) versus $T$ and by introducing $m_{P}$ as the Planck mass, we have obtained the following solution for the black hole temperature

$$
\begin{aligned}
T= & \frac{1}{2 c^{2} \kappa^{2} \alpha_{2}}\left\{8 c \kappa \pi G M\left(1+\sqrt{\alpha_{1} \alpha_{2}}\right)\right. \\
& \left.-\sqrt{-4 c^{2} \kappa^{2} \alpha_{2}\left(c^{4}(1+\chi)+16 G^{2} M^{2} \pi^{2} \alpha_{1}\right)+(8 c \kappa \pi G M)^{2}\left(1+\sqrt{\alpha_{1} \alpha_{2}}\right)^{2}}\right\} .
\end{aligned}
$$

With small values of the deformation parameters, the above equation can be written as

$T=\frac{c^{4}(1+\chi)+16 \alpha_{1} \pi^{2} G^{2} M^{2}}{8 c \kappa \pi G M\left(1+\sqrt{\alpha_{1} \alpha_{2}}\right)}$.

If we consider the absence of correction due to EGUP i.e. $\alpha_{1}=\alpha_{2}=\chi=0$, the above relationship for temperature can be rewritten as

$T=\frac{c^{3}}{8 \kappa \pi G M}=\frac{\left(m_{P} c\right)^{2}}{8 \kappa \pi M}$.

where the relationship of the Planck mass is defined as $\left(m_{P} c\right)^{2}=\frac{c^{3}}{G}$. We can see that the result obtained for the corrected temperature given in Eq. (3.6) in the condition
For small values of deformation parameters, the above equation can be written as

$C=-\frac{c^{3} \kappa\left(c^{2}(1+\chi)+\kappa^{2}(-2+T) T \alpha_{2}\right)}{4 G \pi\left(-(1+\chi) \alpha_{1}+\kappa^{2} T^{2}\left(2-\alpha_{1} \alpha_{2}+2 \sqrt{\alpha_{1} \alpha_{2}}\right)\right)}$,

and it can be seen that the above relationship, for the value of $\alpha_{1}=\alpha_{2}=\chi=0$ in the absence of corrections due to EGUP can be rewritten as

$C=-\frac{c^{5}}{8 \pi \kappa G T^{2}}$.

Therefore, the corrected heat capacity in the absence of EGUP is consistent with the semiclassical heat capacity $C=-\frac{m_{p}{ }^{2} c^{4}}{8 \pi \kappa T^{2}}$ obtained in Refs. [29] and [41].

In order to calculate the entropy $S$ in terms of the black hole mass, a useful heuristic method for a particle which is captured by the black hole has been presented in Ref. [21]. In this method, the black hole is considered as a sink for particles as well as the black hole's size and temperature are denoted by $\Delta X$ and $\Delta P$ parameters respectively. We should consider the fact that the black hole mass is not allowed to be less than a scale order Planck mass and the momentum uncertainty is not allowed to be greater than the mass $(\Delta P \leqslant m)$. When the particle disappears in the black hole horizon, its information is lost to an observer outside the horizon and the loss of information is one bit at least, i.e. $(\Delta S)_{\min } \sim \ln 2$ [21]. Also the smallest increase in the area of a black hole is given by

$A \sim X m$,

where $X$ and $m$ denote the particle's size and mass, respectively. For a classical particle, $(\Delta A)_{\min }=0$, whereas in quantum mechanics, a particle is described by a wave packet. By defining the width of a wave packet as the standard deviation of $X$ distribution, we can consider the position uncertainty as the characteristic size of the particle $(X \sim \Delta X)$. 
Therefore, the representation (3.13) rewrite as follows [21]

$A \geqslant \Delta X \Delta P \geqslant \xi \hbar^{\prime}$,

where $\xi$ and $\hbar^{\prime}$ denote the calibration factor and the effective Planck constant respectively. In order to obtain the effective Planck constant, $\hbar^{\prime}$ in Eq. (3.14), we rewrite Eq. (2.4), which corresponds to the uncertainty relation that arises from EGUP, as

$$
\begin{aligned}
\Delta X \Delta P \geqslant & \frac{1}{2\left(1+\sqrt{\alpha_{1} \alpha_{2}}\right)} \\
& \times\left(1+\chi+\alpha_{1}(\Delta X)^{2}+\alpha_{2}(\Delta P)^{2}\right)=\frac{\hbar^{\prime}}{2} .
\end{aligned}
$$

Therefore, by considering the minimal length in position and momentum uncertainties obtained as Eq. (2.5), the effective Planck constant can be calculated as

$\hbar^{\prime}=\frac{1}{\left(1+\sqrt{\alpha_{1} \alpha_{2}}\right)}\left(1+\chi+\frac{2 \alpha_{1} \alpha_{2}(1+\chi)}{1+2 \sqrt{\alpha_{1} \alpha_{2}}}\right)$.

In terms of the black hole horizon area $A=4 \pi r_{s}^{2}=$ $16 \pi\left(\frac{G^{2} M^{2}}{c^{4}}\right)$, the semiclassical entropy for the Schwarzschild black hole is $S=\frac{A \kappa}{4 \ell_{P}^{2}}$ where $\ell_{P}$ denote the Planck length. In [41], the entropy for the Schwarzschild black hole for ( $\hbar=c=G=\kappa=1$ ) is defined as $S=\frac{A}{4}$. Also, from consistency of thermodynamic information of the black hole in absence of EGUP with the semi-classical case, we can define $\xi=4 \ln 2$ [21]. Also by considering the Heisenberg uncertainty principle, the minimum increase in the horizon area is given by

$\frac{d A}{d S}=\frac{(\Delta A)_{\min }}{(\Delta S)_{\min }}=4 \hbar^{\prime}$.

Therefore, the EGUP-corrected entropy of the black hole can be derived as

$S=\int \frac{d S}{d A} d A \simeq \int \frac{(\Delta S)_{\min }}{(\Delta A)_{\min }} d A \simeq \int \frac{d A}{4 \hbar^{\prime}}$.

From Eqs. (3.16) and (3.17), the corrected entropy can be written in the form

$S=\frac{4 \pi G^{2} M^{2}}{c^{4}}\left(1+\sqrt{\alpha_{1} \alpha_{2}}\right)\left(1+\chi+\frac{2 \alpha_{1} \alpha_{2}(1+\chi)}{1+2 \sqrt{\alpha_{1} \alpha_{2}}}\right)^{-1}$.

For values of $\alpha_{1}=\alpha_{2}=\chi=0$, in the absence of correction due to EGUP, we have

$S=\frac{4 \pi G^{2} M^{2}}{c^{4}}=\pi r_{s}^{2}$.

We can see in the absence of correction due to EGUP, the relationship obtained for entropy reduces to the semiclassical Bekenstein-Hawking entropy for the Schwarzschild black hole as $S=\frac{A}{4}$ [41]. Also we can investigate the behavior of the corrected black hole entropy versus different characteristics of Schwarzschild black hole.

\section{Thermodynamics of Reissner-Nordström black hole}

In this section, we consider a RN spherical black hole with mass $M$ and charge $Q$ described by the metric [44,45]

$d s^{2}=f(r) d t^{2}-\frac{d r^{2}}{f(r)}-r^{2}\left(d \theta^{2}+\sin ^{2} \theta d \varphi^{2}\right)$,

where

$f(r)=1-\frac{2 M}{r}+\frac{Q^{2}}{r^{2}}$.

This metric admits two possible horizons:

$r=M \pm \sqrt{M^{2}-Q^{2}}$.

Actually, when a black hole becomes charged, the event horizon shrinks and another event horizon appears near the singularity and the above two values for $r$ are corresponding to the outer and inner horizons. Since, in the limit of $Q=0$, we should recover the Schwarzschild radius $r_{s}=2 G M / c^{2}$, we consider the plus sign in Eq. (4.3) and the corresponding radius will be radius of the outer horizon.

By defining the radius of the horizon of RN black hole as $r_{R N}=\frac{G r_{0}}{c^{2}}[41]$ where $r_{0}=M+\sqrt{M^{2}-Q^{2}}$, and since near the horizon of the RN black hole, the position uncertainty of a particle will be of the order of RN radius of the black hole, then we find [21]

$\Delta X=\gamma r_{R N}=\frac{\gamma G\left(M+\sqrt{M^{2}-Q^{2}}\right)}{c^{2}}$,

where $\gamma$ is a scale factor. By substituting the value of $\Delta X$ and $\Delta P$ from Eqs. (4.4) and (2.6), the relationship of EUP (2.4) can be rewritten as

$$
\begin{aligned}
r_{0} \geqslant & \frac{\left(m_{p} c\right)^{2}}{2 \gamma \kappa T}\left[1+\chi+\alpha_{1}\left(\frac{\gamma G r_{0}}{c^{2}}\right)^{2}\right. \\
& \left.+\alpha_{2}\left(\frac{\kappa T}{c}\right)^{2}-2 \sqrt{\alpha_{1} \alpha_{2}}\left(\frac{\gamma G r_{0} \kappa T}{c^{3}}\right)\right] .
\end{aligned}
$$

In the absence of correction due to EGUP, from this equation, the black hole temperature can be written as $T=\frac{\left(m_{p} c\right)^{2}}{2 \gamma \kappa r_{0}}$ and comparing this relation with the semiclassical Hawking temperature $T_{H}=\frac{\left(m_{p} c\right)^{2}\left(M r_{0}-Q^{2}\right)}{2 \pi \kappa r_{0}^{3}}$, the value of $\gamma$ is seen to be

$\gamma=\frac{\pi r_{0}^{2}}{M r_{0}-Q^{2}}$.

This result is consistent with the obtained result in the absence of correction due to GUP [41]. By the identity $M r_{0}-Q^{2}=$ $r_{0}\left(r_{0}-M\right)$, and inserting Eq. (4.6) into Eq. (4.5), we obtain 
the following mass-charge-temperature relation corresponding to the RN black hole:

$$
\begin{aligned}
T= & \frac{1}{\kappa^{2} m_{p}{ }^{4}\left(M-r_{0}\right)^{2} \alpha_{2}}\left\{\kappa m_{p}{ }^{2} \pi\left(r_{0}-M\right) r_{0}^{2}\left(1+\sqrt{\alpha_{1} \alpha_{2}}\right)\right. \\
& \left.\left.-\sqrt{-\kappa^{2} m_{p}{ }^{4}\left(M-r_{0}\right)^{2}\left(c^{2} m_{p}{ }^{4}\left(M-r_{0}\right)^{2}(1+\chi) \alpha_{2}-\pi^{2} r_{0}{ }^{4}\left(1+2 \sqrt{\alpha_{1} \alpha_{2}}\right)\right.}\right)\right\} .
\end{aligned}
$$

From the identity $M r_{0}-Q^{2}=r_{0}\left(r_{0}-M\right)$, we get for small values of deformation parameters :

$$
T=\frac{\left(m_{p} c\right)^{2}\left(r_{0}-M\right)(1+\chi)\left(1+\sqrt{\alpha_{1} \alpha_{2}}\right)}{2 \pi \kappa r_{0}^{2}\left(1+2 \sqrt{\alpha_{1} \alpha_{2}}\right)} .
$$

With $\alpha_{1}=\alpha_{2}=\chi=0$ in the absence of correction due to EGUP, we have

$$
T=\frac{\left(m_{p} c\right)^{2}\left(r_{0}-M\right)}{2 \pi \kappa r_{0}^{2}} .
$$

This equation is consistent with the usual semiclassical Hawking temperature in the absence of correction due to GUP [41]. Now the heat capacity of the RN black hole can be calculated as

$$
\begin{aligned}
C= & c^{2} \frac{d M}{d T}=\left(\frac{1}{c^{2}} \frac{d T}{d M}\right)^{-1} \\
= & \left(c^{2} \kappa^{2} m_{p}{ }^{4}\left(M^{2}-Q^{2}\right)^{2} \alpha_{2}\right)\left\{-2 M \kappa m_{p}^{2} \pi\right. \\
& \sqrt{M^{2}-Q^{2}}\left(M+\sqrt{M^{2}-Q^{2}}\right)^{2}\left(1+\sqrt{\alpha_{1} \alpha_{2}}\right)+2 M \mu \\
& +\frac{1}{2} \kappa m_{p}^{2}\left(M^{2}-Q^{2}\right)\left[4 \pi\left(M+\sqrt{M^{2}-Q^{2}}\right)^{2}\right. \\
& \left(1+\sqrt{\alpha_{1} \alpha_{2}}\right)+\frac{2 M \pi\left(M+\sqrt{M^{2}-Q^{2}}\right)^{2}\left(1+\sqrt{\alpha_{1} \alpha_{2}}\right)}{\sqrt{M^{2}-Q^{2}}} \\
& -\frac{\kappa m_{p}^{2}}{\mu}\left(-2 M c^{2} m p^{4}\left(M^{2}-Q^{2}\right)(1+\chi) \alpha 2\right. \\
& \left.+2 M \pi^{2}\left(M+\sqrt{M^{2}-Q^{2}}\right)^{4}(1+2 \sqrt{\alpha 1 \alpha 2})\right) \\
& +\frac{\kappa m_{p}{ }^{2}}{\mu}\left(M^{2}-Q^{2}\right)\left(2 c^{2} M m p^{4}(1+\chi) \alpha 2\right. \\
& \left.\left.-\frac{4 \pi^{2}\left(M+\sqrt{M^{2}-Q^{2}}\right)^{4}(1+2 \sqrt{\alpha 1 \alpha 2})}{M^{2}-Q^{2}}\right]\right\}^{-1},
\end{aligned}
$$

where

$$
\begin{aligned}
\mu= & \left(\left(\kappa m_{p}{ }^{2}\right)^{2} \pi^{2} r_{0}^{4}\left(1+2 \sqrt{\alpha_{1} \alpha_{2}}\right)\left(r_{0}-M\right)^{2}\right. \\
& \left.-\left(\kappa m_{p}{ }^{4}\right)^{2} c^{2}\left(r_{0}-M\right)^{4}(1+\chi) \alpha_{2}\right)^{\frac{1}{2}} .
\end{aligned}
$$

If we take into account Eq. (4.3), the heat capacity of the RN black hole can be rewritten as

$$
\begin{aligned}
C= & \left(c^{2} \kappa^{2} m_{p}^{4}\left(r_{0}-M\right)^{4} \alpha_{2}\right) \\
& \times\left\{-2 M \kappa m_{p}^{2} \pi\left(r_{0}-M\right) r_{0}^{2}\left(1+\sqrt{\alpha_{1} \alpha_{2}}\right)+2 M \mu\right. \\
& +2 \kappa m_{p}^{2} \pi r_{0}^{2}\left(1+\sqrt{\alpha_{1} \alpha_{2}}\right)\left(r_{0}-M\right)^{2} \\
& +\kappa m_{p}^{2} M \pi r_{0}^{2}\left(1+\sqrt{\alpha_{1} \alpha_{2}}\right)\left(r_{0}-M\right) \\
& +\frac{2\left(\kappa m_{p}^{4}\right)^{2}}{\mu} M c^{2}\left(r_{0}-M\right)^{4}(1+\chi) \alpha_{2} \\
& -\frac{\left(\kappa m_{p}^{2}\right)^{2}}{\mu} M \pi^{2} r_{0}^{4}\left(1+2 \sqrt{\alpha_{1} \alpha_{2}}\right)\left(r_{0}-M\right)^{2} \\
& \left.-\frac{\left(\kappa m_{p}^{2}\right)^{2}}{\mu} 2 \pi^{2} r_{0}^{4}\left(1+2 \sqrt{\alpha_{1} \alpha_{2}}\right)\left(r_{0}-M\right)^{3}\right\}^{-1} .
\end{aligned}
$$

For small values of deformation parameters and in the absence of correction due to EGUP, the obtained heat capacity for the charged black hole is consistent with results obtained previously in the absence of correction due to GUP [41].

In order to obtain the entropy $S$ in terms of the mass of the black hole $M$, we have used the method presented in the previous section for a particle which is absorbed by the black hole [16]. By considering the RN black hole as a sink for absorbing particles and the black hole's size and temperature via $\Delta X$ and $\Delta P$ parameters, the smallest increase in the area of a black hole is given by

$A \sim X m \geqslant \Delta X \Delta P \geqslant \xi \hbar^{\prime}$.

By considering the effective Planck constant $\hbar^{\prime}$ introduced in Eq. (3.16), the EGUP-corrected entropy of the static and spherical charged black hole can be expressed as

$$
\begin{aligned}
S= & \int \frac{d S}{d A} d A \simeq \int \frac{(\Delta S)_{\min }}{(\Delta A)_{\min }} d A \simeq \int \frac{d A}{4 \hbar^{\prime}} \\
= & \frac{\pi G^{2}}{c^{4}}\left(1+\sqrt{\alpha_{1} \alpha_{2}}\right)\left(M+\sqrt{M^{2}-Q^{2}}\right)^{2} \\
& \times\left(1+\chi+\frac{2 \alpha_{1} \alpha_{2}(1+\chi)}{1+2 \sqrt{\alpha_{1} \alpha_{2}}}\right)^{-1} .
\end{aligned}
$$

As expected, in the limit of $Q=0$, the RN black hole entropy is recovered to the calculated entropy of the Schwarzschild black hole in Eq. (3.19). Also we can see in the absence of correction due to EGUP for $\alpha_{1}=\alpha_{2}=\chi=0$ and by setting 
the specific constants $c, \kappa$ and $G$ as unity, Eq. (4.14) reduces to the semi-classical entropy for the black hole $S=\frac{A}{4}$ [41].

\section{Conclusions}

In this paper, we have investigated thermodynamical properties of the Schwarzschild and RN black holes by taking in account the EGUP. The lower bound for the EGUP black hole temperature has been obtained by the minimal momentum of EGUP. We have calculated the mass-temperature relation for the Schwarzschild black hole with EGUP and the masstemperature-charge relation for EGUP RN black hole. We have calculated the EGUP-corrected entropy in terms of mass for the Schwarzschild black hole and in terms of mass and charge for the RN black hole, respectively. We examined the behaviour of the corrected entropy versus the black hole's mass. Also, we calculated the corrected heat capacity for the Schwarzschild and charged black holes. Moreover, the results obtained for the thermodynamical properties of two black holes in this work have been compared with each other and with previous standard forms already given in the literature.

Acknowledgements The authors thank the referee for a thorough reading of our manuscript and for constructive suggestions. MdeM is grateful to the Natural Sciences and Engineering Research Council (NSERC) of Canada for partial financial support (Grant number RGPIN-201604309).

Data Availability Statement This manuscript has no associated data or the data will not be deposited. [Authors' comment: In this paper, we investigate thermodynamic properties of the Schwarzschild and RN black holes under the consideration of EGUP, by calculating the EGUP corrected temperature, entropy and heat capacity vs. black hole's characteristics. However, this manuscript has no associated data.]

Open Access This article is distributed under the terms of the Creative Commons Attribution 4.0 International License (http://creativecomm ons.org/licenses/by/4.0/), which permits unrestricted use, distribution, and reproduction in any medium, provided you give appropriate credit to the original author(s) and the source, provide a link to the Creative Commons license, and indicate if changes were made. Funded by SCOAP ${ }^{3}$.

\section{References}

1. C. Bambi, F.R. Urban, Class. Quant. Grav. 25, 095006 (2008)

2. A.F. Ali, Class. Quant. Grav. 28, 065013 (2011)

3. A.F. Ali, S. Das, E.C. Vagenas, Phys. Rev. D 84, 044013 (2011)

4. S. Das, E.C. Vagenas, A.F. Ali, Phys. Lett. B 690, 407 (2010)
5. S. Das, E.C. Vagenas, A.F. Ali, Phys. Lett. B 692, 342 (2010)

6. A.F. Ali, JHEP 1209, 067 (2012)

7. H.S. Snyder, Phys. Rev. 71, 38 (1947)

8. D.J. Gross, P.F. Mende, Nucl. Phys. B 303, 407 (1988)

9. D. Amati, M. Ciafaloni, G. Veneziano, Phys. Lett. B 216, 41 (1989)

10. A. Kempf, G. Mangano, R.B. Mann, Phys. Rev. D 52, 1108 (1995)

11. M. Kato, Phys. Lett. B 245, 43 (1990)

12. F. Scardigli, Phys. Lett. B 452, 39 (1999)

13. J. Magueijo, L. Smolin, Phys. Rev. Lett. 88, 190403 (2002)

14. J. Magueijo, L. Smolin, Phys. Rev. D 71, 026010 (2005)

15. J.L. Cortes, J. Gamboa, Phys. Rev. D 71, 065015 (2005)

16. H. Hassanabadi, E. Maghsoodi, Won Sang Chung, Eur. Phys. J. C 79, 358 (2019)

17. A.F. Ali, S. Das, E.C. Vagenas, Phys. Lett. B 678, 497 (2009)

18. Won Sang Chung, H. Hassanabadi, Min Su Kang, Hee Hun Sung, J. Maeng, S. Lee, Young Il Moon, Mod. Phys. Lett. A 33, 1950052 (2019)

19. G. Amelino-Camelia, Int. J. Mod. Phys. D 11, 35 (2002)

20. G. Amelino-Camelia, Phys. Lett. B 510, 255 (2001)

21. Li Xiang, X.Q. Wen, JHEP 0910, 046 (2009)

22. J.L. Cortes, J. Gamboa, Phys. Rev. D 71, 065015 (2005)

23. B. Bolen, M. Cavagliá, Gen. Relativ. Grav. 37, 1255 (2005)

24. S. Mignemi, Mod. Phys. Lett. A 25, 1697 (2010)

25. T. Zhu, J.R. Ren, M.F. Li, Phys. Lett. B 674, 204 (2009)

26. S. Hossenfelder, Living Rev. Rel. 16, 2 (2013)

27. B. Ji-long, W. Ting-dun, Chin. Astron. Astrophys. 39, 447 (2015)

28. Won Sang Chung, H. Hassanabadi, Mod. Phys. Lett. A 32, 26 (2017)

29. Won Sang Chung, H. Hassanabadi, Phys. Lett. B 793, 451 (2019)

30. J.R. Mureika, Phys. Lett. B 789, 88 (2019)

31. G. Veneziano, Proceedings of PASCOS 90, Quantum Gravity near the Planck scale, Boston 1990, p. 486 (unpublished)

32. M. Falek, M. Merad, M. Moumni, J. Math. Phys. 60, 013505 (2019)

33. S. Mignemi, Class. Quantum Gravit. 29, 215019 (2012)

34. M.M. Stetsko, J. Math. Phys. 56, 012101 (2015)

35. S. Capozziello, G. Lambiase, G. Scarpetta, Int. J. Theor. Phys. 39, $15(2000)$

36. R.J. Adler, P. Chen, D.I. Santiago, Gen. Rel. Grav. 33, 2101 (2001)

37. A. Medved, E.C. Vagenas, Phys. Rev. D 70, 124021 (2004)

38. Y.S. Myung, Y.-W. Kim, Y.-J. Park, Phys. Lett. B 645, 393 (2007)

39. K. Nouicer, Phys. Lett. B 646, 63 (2007)

40. A.F. Ali, H. Nafie, M. Shalaby, Europhys. Lett. 100, 20004 (2012)

41. S. Gangopadhyay, A. Dutta, Advances in High Energy Physics Volume 2018, Article ID 7450607, 9 pages

42. K. Nozari, B. Fazlpour, Acta Phys. Polon. B 39, 1363 (2008)

43. Y. Kumaran, A. Ovgun, arXiv:1905.11710v1

44. H. Reissner, Annalen der Physik 355, 106 (1916)

45. G. Nordström, Proc. Kon. Ned. Akad. Wet. 20, 1238 (1918) 